\title{
Hepatitis B Infection and Immunity among Pregnant Women Attending Antenatal Clinics in Health Centers of Mbarara Municipality, Southwestern Uganda
}

\author{
Immaculate Kabajulizi, Joel Bazira, Collins Atuheire, Charles Kato, Taseera Kabanda \\ Department of Microbiology, Mbarara University of Science and Technology, Mbarara, Uganda \\ Email:kmmaculate@yahoo.com
}

How to cite this paper: Kabajulizi, I., Bazira, J., Atuheire, C., Kato, C. and Kabanda, T. (2019) Pregnant Women Attending Antenatal Clinics in Health Centers of Mbarara Municipality, Southwestern Uganda. Advances in Infectious Diseases, 9, 65-79.

https://doi.org/10.4236/aid.2019.92006

Received: April 29, 2019

Accepted: May 24, 2019

Published: May 27, 2019

Copyright $\odot 2019$ by author(s) and Scientific Research Publishing Inc. This work is licensed under the Creative Commons Attribution International License (CC BY 4.0).

http://creativecommons.org/licenses/by/4.0/

\section{Open Access}

\begin{abstract}
Introduction: Viral hepatitis B (HBV) in pregnancy is a risk for childhood transmission where the majority become chronically infected. In Uganda, $\mathrm{HBV}$ is not tested for during antenatal, therefore the number of infected, infectious, immune and none-immune pregnant women is unknown curtailing efforts to prevent mother to child transmission. Methods: We conducted a descriptive cross-sectional study involving 254 pregnant women from four health centers in Mbarara Municipality. HBV status was assessed using an immunochromatographic (COMBO) kit, the type of infection; based on demonstration of anti hepB core IgM (acute infection) and total core IgG antibodies (chronic infection) and infectiousness; based on the presence of $\mathrm{HBeAg}$ and/or a quantitative $\mathrm{HBV}$ viral load $\geq 20,000 \mathrm{IU} / \mathrm{mL}$. Immunity was determined using the COMBO kit and HBsAb quantification ELISA. One was deemed immune to $\mathrm{HBV}$ if HBsAb titers were $\geq 10 \mathrm{mIU} / \mathrm{mL}$. Results: The prevalence of $\mathrm{HBV}$ infection was $1.2 \% ; 33 \%$ and $67 \%$ with acute and chronic HBV respectively. 33\% were infectious based on a high viral load, none had detectable $\mathrm{HBeAg}$. 14\% were immune; amongst whom $72 \%$ had natural exposure and $18 \%$ after vaccination. There was insufficient immunity in $11 \%$ with a majority (75\%) having acquired immunity following vaccination. Conclusion: The prevalence of $\mathrm{HBV}$ is low and most of those are chronically infected. HBeAg and Hepatitis B viral load should be performed when evaluating infectiousness. Further, there is a high transmission of HBV among adults and a low uptake of the HBV vaccine in Mbarara Municipality.
\end{abstract}

\section{Keywords}

Hepatitis B Virus, Prevalence, Acute and Chronic, Viral Load, Immunity, 
Pregnant Women

\section{Background}

Hepatitis B virus causes morbidity and mortality especially among persons that are chronically infected [1]. The risk for chronic hepatitis B infection is largely dependent on the age of acquisition of the virus; transmission to neonates and children below 5 years result in $90 \%$ of them developing chronic Hepatitis B [2] due to inability of the body to mount sufficient immunity to eradicate the virus [3]. Pregnant women are a major source of infection to children from whom transmission occurs during pregnancy, at birth or early infancy through contact with various body fluids [4].

Infectiousness of Hepatitis B or risk of transmission depends on replication of the virus which is evidenced by the presence of HBeAg or Hepatitis B viral load $\geq 20,000 \mathrm{IU} / \mathrm{mL}$ [5]. Therefore, in order to prevent this mother-to-child transmission, there is a need to identify pregnant women who are infected as well as those that are infectious so that preventive measures are instituted during and shortly after birth.

At present observation of the Uganda antenatal integrated register Health management information system (HMIS) form 071 shows that there is no routine testing for Hepatitis B during pregnancy as it is for other infections like HIV and Syphilis, yet routine vaccination against hepatitis was not publically available until 2012 under the Uganda National Extended Program on Immunization (UNEPI) [6]. Thus, the magnitude of infection among pregnant women is unknown, and no specific preventive measures are instituted before, during and after birth. Data from the Uganda Population-Based HIV Impact Assessment (UPHIA) put the Country's prevalence of Hepatitis B virus at 4.1\% [7]. Unfortunately, this surveillance report is silent on the prevalence of Hepatitis B among pregnant women; moreover, there is evidence of the infection being particularly higher among pregnant women than in the general population. For instance, in a study done among 379 pregnant women in 2 regional referral hospitals, Gulu and Lacor hospitals both were located in northern Uganda, the prevalence of hepatitis B was $11.8 \%$, which was higher than the $4.6 \%$ in the mid-northern region [7] and $14.9 \%$ of these women were potentially infectious based on detection of a positive Hepatitis B envelop antigen (HBeAg) [8]. There was a likelihood that this study under-reported the potential infectiousness of these pregnant women. Recent studies have shown patients in whom the HBeAg is negative yet they have a high Hepatitis B viral load $\geq 20,000 \mathrm{IU} / \mathrm{mL}$ [9] and thus infectious.

The ability of the body to protect itself from infection or immunity [10] can be acquired artificially through vaccination) or naturally after recovery from infection. In both scenarios, the individual develops anti-hepatitis B surface antibodies, but these should exceed $10 \mathrm{mlU} / \mathrm{mL}$ for them to be protected from infec- 
tion or reactivation [11]. This study was designed to determine the prevalence of viral Hepatitis B among pregnant women, classify whether the infection was acute or chronic, determine whether the pregnant women were infectious or not and to evaluate immunity among those who were HBV negative.

\section{Methodology}

\subsection{Study Sites}

The study was carried out at four (4) public health centers in Mbarara Municipality that were Mbarara Municipal Council Health Centre IV (MMCHCIV), Biharwe Health Centre III (BHCIII), Nyamitanga Health Center III (NHCIII) and Kakoba Health Center (KHCIII).

\subsection{Study Design and Duration}

This was a descriptive cross-sectional study employing quantitative methods of data collection. Data were collected from September 2018 to November 2018

\subsection{Study Population}

All pregnant women who attended antenatal care (ANC) from the study sites during the study period were considered eligible. The study included consented pregnant women but it excluded those pregnant women who had; high blood pressure $140 / 90 \mathrm{mmHg}$, bleeding from the vagina and axillary body temperature above $38.3^{\circ} \mathrm{C}$. These pregnant women, however, were to be included if they recovered and visited ANC at the selected health facilities within the sample collection period.

\subsection{Sample Size Estimation (Formula and Assumption)}

The sample size was calculated based on Kish-Leslie's formulae for cross-sectional studies [12]. The prevalence of $19.4 \%$ was used where $9.4 \%$ was calculated from $11.8 \%$ prevalence of Hepatitis B among pregnant women in Northern Uganda [8]. This was multiplied by $0.8 \%$ prevalence of Hepatitis B in Southwestern Uganda [7]. We added $10 \%$ since pregnant women were engaged in unprotected sex, a known risk factor for STIs compared with the general population [13] and we added $10 \%$ of the calculated sample size to cater for attrition and missing data. We enrolled 254 pregnant women.

\subsection{Sampling Procedure}

We used stratified sampling in which the four study sites were designated as an individual stratum. We applied proportionately by probability based on the average number of pregnant women seen at ANC each day at every Health Center. This was determined by dividing the number of pregnant women who had attended ANC at each Health Centre in the past one week (before the study) by the total number that attended ANC at all the four health units in that week and multiplied this by the desired sample size (254). Participants were enrolled 
through consecutive sampling for a period of one week at each facility.

\subsection{Recruitment Procedure}

Figure 1: In this study, we screened a total of 264 pregnant women from the antenatal clinics of Four Lower Health facility; 1) Mbarara Municipal Council Health Center IV (MMCHCIV), 2) Biharwe Health Center III (BHCIII), 3) Nyamitanga Health Center III (NHCIII) and 4) Kakoba Health Center III (KHCIII). We excluded 10 pregnant women because; 2 had vaginal bleeding, 2 had a high blood pressure $\geq 140 / 90 \mathrm{mmHg}, 3$ had a high body temperature $\geq$ $38.3^{\circ} \mathrm{C}$ and 3 declined to participate. Therefore, this study enrolled 254 pregnant women.

\subsection{Study Procedures}

As part of the consent process, pregnant women were informed about Hepatitis $B$; its transmission, symptoms and prevention, the study purpose and procedures after the Health talk at the Antenatal waiting area. Consenting was carried out by RAs who were trained counselors. Thereafter consented participants were subjected to an interviewer guided questionnaire which captured information on social demographics, vaccination status, and clinical history. This was followed by a blood draw where a maximum of $5 \mathrm{~mL}$ of Blood was collected into two vacutainers (plain and EDTA tubes) for protocol approved tests as well as other routine ANC tests using either 21 or 23-gauge needle. The samples collected in the plain vacutainers were allowed to stand at room temperature for 5 minutes in order for coagulation to take place. The coagulated blood was centrifuged at $3000 \mathrm{rpm}$ for 5 minutes from which we harvested $1 \mathrm{~mL}$ of serum and transferred

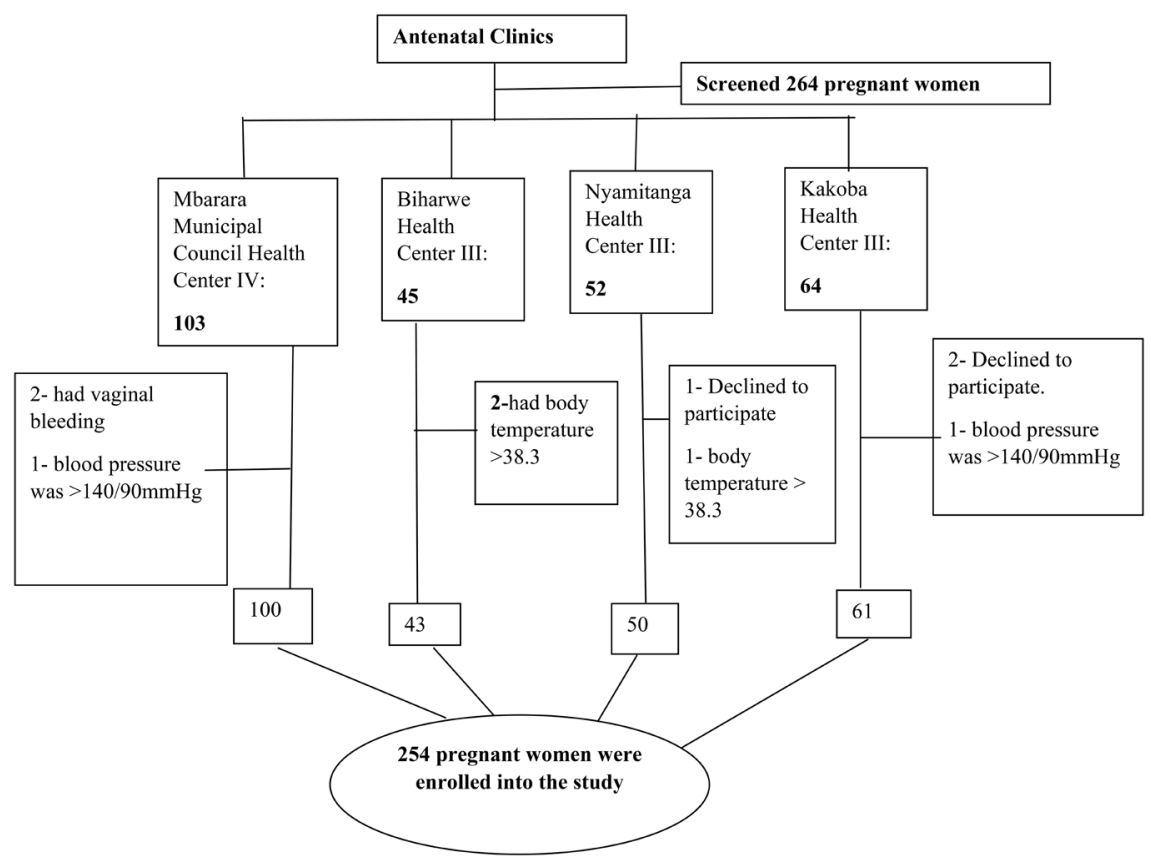

Figure 1. The recruitment procedure. 
it into cryo vials; this was used for ALT and AST determination and afterwards storage for later assays like anti-Hepatitis B core IgM and HBsAb quantification tests. The blood samples in EDTA vacutainers were used to first perform the regular ANC laboratory tests i.e. HIV, syphilis, or blood grouping as requested by the site Clinician. Those samples were then centrifuged at a speed of 3000 rpm for 3 minutes toobtain $1 \mathrm{~mL}$ of plasma. From the plasma, $300 \mu \mathrm{l}$ were put on a COMBO 5 in 1 test kit (Vaxpert, USA) to test for HBsAg, HBsAb, HBeAg, $\mathrm{HBeAb}$ and $\mathrm{HBcAb}$. The remaining plasma approximately $0.7 \mathrm{~mL}$ was transferred into cryo vials and stored at $-20^{\circ} \mathrm{C}$ for later determination of Hepatitis $\mathrm{B}$ viral load. Laboratory results were categorized into four based on the reaction on the COMBO 5 in 1 kit. The categories were: 1) At risk; these had a negative reaction on all the 5 parameters of a COMBO 5 in 1 kit. 2) Immune to $\mathrm{HBV}$ due to natural exposure; these had a negative $\mathrm{HBsAg}$ and $\mathrm{HBeAg}$ while positive $\mathrm{HBsAb}$ and $\mathrm{HBcAb}, 3)$ or Immune to $\mathrm{HBV}$ due to artificial vaccination; these had negative $\mathrm{HBsAg}, \mathrm{HBeAg}, \mathrm{HBeAb}$ and $\mathrm{HBcAb}$ but positive for $\mathrm{HBsAb}$, 4) or Hepatitis $B$ positive; who had positive $\mathrm{HBsAg}, \mathrm{HBcAb}$, but Negative HBsAb. Plasma and serum aliquots were taken to MMCHCIV laboratory for storage at $-20^{\circ} \mathrm{C}$ and for analysis of liver enzymes namely Alanine aminotransferase (ALT) and Aspartate aminotransferase (AST) using Cobas c111 clinical chemistry analyzer (Roche-Germany). Hepatitis B positive samples were shipped to MBN clinical laboratories where $100 \mu \mathrm{l}$ of serum was used to perform qualitative HBcAb IgM, $50 \mu$ to perform quantitative HBsAb ELISA technique and identified those participants with enough immunity ( $\mathrm{HBsAb} \geq 10 \mathrm{mIU} / \mathrm{ml}$ ) and those who had low immunity ( $\mathrm{HBs} \mathrm{Ab}<10 \mathrm{mIU} / \mathrm{ml}$ ).

In addition, $0.7 \mathrm{~mL}$ of plasma aliquot was taken to Uganda Health Laboratory services (UNHLS) in Luzira-Kampala where $500 \mu \mathrm{l}$ of plasma was used to determine the number of Hepatitis B viral copies using Polymerase Chain Reaction technique (PCR) on CobasAmpliPrepTaqman machine; (Roche-Germany). A participant was deemed infectious when the blood sample contained HBeAg and/or viral load $\geq 20,000 \mathrm{IU} / \mathrm{mL}$.

Results were recorded on a result slip indicating all the 5 parameters on the COMBO kit as either positive or negative, participants' name, study number and category. Results from the Hepatitis B screening test were released within one hour of blood draw and were communicated to the individual participants after post-test counseling. Other results (ALT/AST, ELISA tests and Viral load) were delivered to midwives at ANCs of the respective facilities after a period of one month; the midwives were training on how to release results. Since Hep B is infectious, HBsAg positive participants were requested to inform their spouses and bring them on their day of convenience for Hep B counseling and testing. However, those participants who found it difficult to inform their spouses, we offered Assisted Partner Notification (APN) based on the model used for HIV. Those participants' spouses who were found HBV positive were referred to as an Infectious Diseases' Physician for further management. Pregnant women who were 
identified as infectious based on a Hepatitis B viral load $>20,000 \mathrm{IU} / \mathrm{mL}$ and/or with a Positive HBeAg were contacted and referred to a specialized team composed of an Infectious Disease Specialist, Obstetrician and a Paediatrician who ensured the wellbeing of the mother and the newborn. All newborn babies were vaccinated within 24 hours of birth.

\subsection{Data Analysis}

Data were entered into a laboratory work list, and thereafter transferred into Microsoft Excel then imported into STATA version 12 (Statacorp, 4905 Lakeway Drive, College Station, Texas 77845, USA) for analysis. Socio-demographic and clinical factors were analyzed descriptively as means (standard deviation), for continuous variables, and frequencies, percentages ( $95 \%$ CI) for categorical variables. Age was categories into two; 20 years and below, and above 20 years. Because according to the study among pregnant women in Uganda, it was found that HBsAg was prevalent among the age of 20 years and below [8]. The Chi square was used to compare results between age categories, health facilities and HIV status. Data presentation was done using tables and figures.

\subsection{Ethical Considerations}

This study was of high risk in a way that it triggered psychological discomfort and physical discomfort due to anxiety about additional tests to those normally carried out for ANC. We, however, attempted to make it less discomforting by; providing pretest and post-test counselling. The study was approved by Institutional Ethical Review Committee of Mbarara University of Science and permission to collect data from the four health centers was granted by the department of health of Mbarara Municipal Council. Only Consented participants were enrolled.

\section{Results}

\subsection{Demographic Characteristics of Study Participants}

Table 1: There were 254 participants in the study with a mean age of 24.8 years, and a standard deviation (sd) of \pm 4.88 (range 18 - 41 years). Majority of the participants were co-habiting $87.8 \%$ (223/254), with many having attended secondary education level $44.5 \%(113 / 254)$, also many were occupied in different business ventures $44.5 \%(113 / 254)$. Overall the participants were residents of Mbarara district $98.4 \%(250 / 254)$, on the assessment of the HIV status 9\% (23/254) of the participants were HIV positive, however, $99.83 \%$ of the HIV positive participants were on HIV therapy.

\subsection{Prevalence of Hepatitis B Surface Antigen}

Table 2: The prevalence of Hepatitis B surface antigen (HBsAg) was $1.2 \%$ (3/254). The prevalence of HBsAg was not statistically different between those 
participants aged $\leq 20$ and $>20$ years. Among the health centers, the prevalence of HBsAg was highest in BHC III however; there was no statistical difference with the other health centers. Hepatitis B surface antigen was prevalent among HIV negative participants.

\subsection{Type of Hepatitis B Infection (Acute or Chronic)}

Figure 2: Overall 3 participants (1.2\%) were positive for the HBsAg. Of these 1 (33\%) had an acute infection and $2(67 \%)$ had chronic infection.

Table 1. Demographic characteristics.

\begin{tabular}{|c|c|}
\hline Variable & Summary measure \\
\hline \multicolumn{2}{|l|}{ Age in categories $\mathrm{n}(\%)$} \\
\hline 20 and below & $53(20.9)$ \\
\hline Above 20 & $201(79.1)$ \\
\hline \multicolumn{2}{|l|}{ Marital status n (\%) } \\
\hline Legally married & $25(9.84)$ \\
\hline Co-habiting & $223(87.80)$ \\
\hline Separated & $4(1.57)$ \\
\hline Others & $2(0.79)$ \\
\hline \multicolumn{2}{|l|}{ Education n (\%) } \\
\hline None & $10(3.94)$ \\
\hline Primary & $87(34.35)$ \\
\hline Secondary & $113(44.49)$ \\
\hline Tertiary & $44(17.32)$ \\
\hline \multicolumn{2}{|l|}{ Occupation n (\%) } \\
\hline Housewives & $102(40.16)$ \\
\hline Accommodation & $10(3.94)$ \\
\hline Food vendors & $5(1.97)$ \\
\hline Office workers & $4(1.57)$ \\
\hline Waste management & $1(0.39)$ \\
\hline Education services & $18(7.09)$ \\
\hline Military and police & $1(0.39)$ \\
\hline Others (Business not classified) & $113(44.49)$ \\
\hline \multicolumn{2}{|l|}{ District of residence $\mathrm{n}(\%)$} \\
\hline Isingiro & $4(1.57)$ \\
\hline Mbarara & $250(98.43)$ \\
\hline \multicolumn{2}{|l|}{ HIV status n (\%) } \\
\hline Negative & $231(90.94)$ \\
\hline Positive & $23(9.06)$ \\
\hline
\end{tabular}


Table 2. Proportion of pregnant women with positive HBsAg.

\begin{tabular}{ccc}
\hline Variable & Prevalence (\%) & $p$-value \\
\hline Age categories & 1.9 & 0.593 \\
$\leq 20$ & 1 & \\
$>20$ & & 0.749 \\
Health centers & 2.3 & \\
BHC III & 1.6 & \\
KHC III & 1 & 0.582 \\
MMCHC IV & 0 & \\
NHC III & & \\
HIV status & 0 & \\
positive & 1.3 & \\
Negative &
\end{tabular}

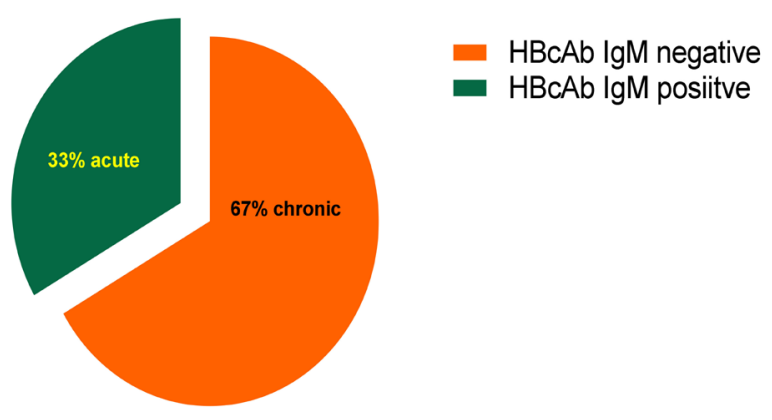

Figure 2. A pie chart showing the type of Hepatitis B infection (acute or chronic).

\subsection{Evaluation of Infectiousness of HBV among HBsAg Positive Participants}

Table 3: The evaluation of infectiousness of HBV was based on the presence of a positive Hepatitis B envelop antigen ( $\mathrm{HBe} A g$ ) and/or a high viral load $>20,000$ $\mathrm{IU} / \mathrm{mL}$. All the $3 \mathrm{HBsAg}$ positive participants were negative for HBeAg and only one had a high viral load. Figure 3: Comparing the viral load among those with acute and chronic Hepatitis B infection, we found that chronically infected individuals had a high viral load.

\subsection{Evaluation of Immunity}

Figure 4: In this study, $14.6 \%$ of the participants were immune to HBV (HBsAb positive) either by artificial vaccination (HBcAb negative) or by natural exposure to $\mathrm{HBV}$ (HBcAb positive) and $84.2 \%$ were none immune (HBsAg and HBsAb negative). Table 4: There was a statistical difference $(p$-value $=0.02)$ between the number of participants who had acquired immunity by natural exposure to HBV (26/36) and those who acquired immunity by artificial vaccination (10/36).

Figure 5: On quantification of HBsAb, 11\% (4/36) had insufficient immunity 
i.e. $\mathrm{HBsAb}$ titers $<10 \mathrm{mIU} / \mathrm{mL}$ while $88 \%(32 / 36)$ had sufficient immunity i.e. $\mathrm{HBsAb} \geq 10 \mathrm{mIU} / \mathrm{mL}$. Comparing Natural and artificial immunity HBsAb titers, $(1 / 26)$ of the naturally exposed participants had insufficient immunity, and 30\% (3/10) of the artificially vaccinated participants had insufficient immunity. Furthermore, the $3 / 10$ artificially vaccinated participants with insufficient immunity; we analyzed for the number of vaccine doses and their individual HBsAb titers, we discovered that $2 / 3$ had completed their vaccination dosage ( 3 doses) and the HBsAb titers were tending to $10 \mathrm{mIU} / \mathrm{mL}$. On the other hand, $1 / 3$ had no knowledge of vaccination.

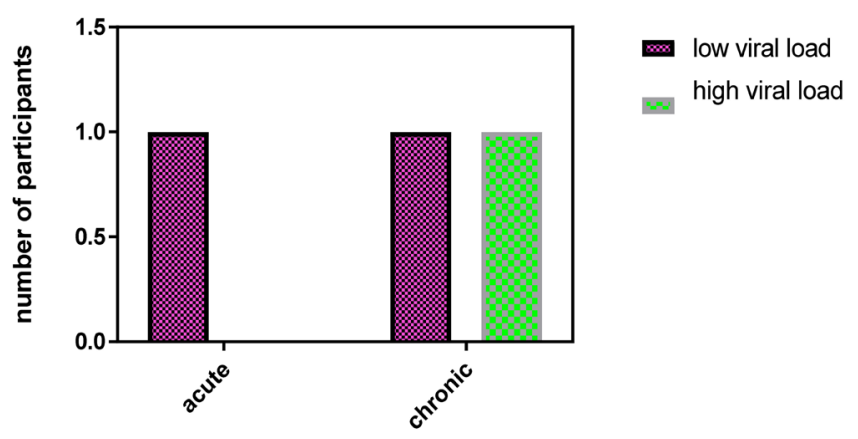

Figure 3. Comparison of viral load based on the type of infection (acute and chronic infection).

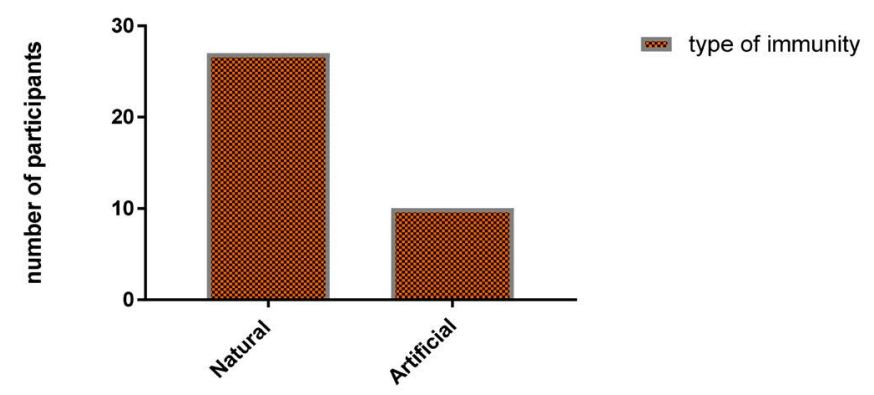

Figure 4. Natural and artificial immunity among pregnant women in health centers' ANCs.

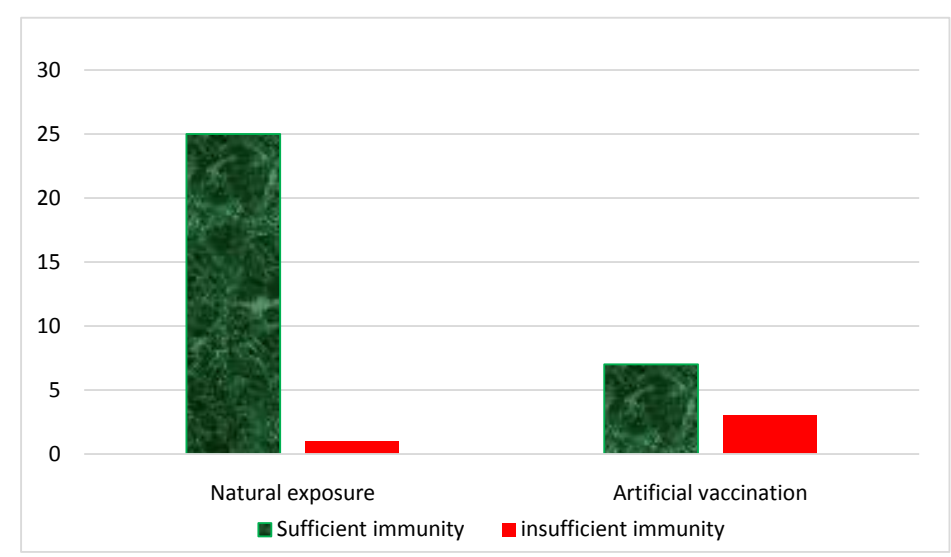

Figure 5. Comparison of HBsAb titers between participants with Natural and artificial immunity. 
Table 3. Evaluation of infectiousness of Hepatitis B virus among HBsAg positive participants.

\begin{tabular}{ccc}
\hline HBeAg & Positive & Negative \\
\hline \multirow{3}{*}{ Viral load } & 0 & 3 \\
& high & low \\
& 1 & 2 \\
\hline
\end{tabular}

Table 4. Type of immunity among pregnant women in health centers' ANCs.

\begin{tabular}{cccc}
\hline Type of immunity & Percentage & $95 \%$ CI & $p$-value \\
\hline Artificial & 28 & $0.12-0.43$ & 0.02 \\
Natural & 72 & $0.57-0.88$ & \\
\hline
\end{tabular}

\section{Discussion}

\subsection{Prevalence of Pregnant Women Positive for Hepatitis B Surface Antigen}

The prevalence of Hepatitis B surface antigen positivity (HepB infection) was $1.2 \%$. This value may be comparable to a recent population survey of Hepatitis $B$ in south western Uganda of $0.8 \%$ [7]. However, in the 2017 survey, a larger sample size was used and they studied a general population. Our findings are much lower than the $11.8 \%$ among pregnant women in Northern Uganda [8] this may be due to the regional differences in the prevalence of HBV between southwest $(0.8 \%)$ and Mid-northern Uganda (4.6\%) [7]. The prevalence in our study was lower than $3 \%$ of the country prevalence of hepatitis B in women aged $16-64$ [7]. Hence this is reflective of the fact that Hepatitis B prevalence in Southwestern Uganda is still low.

\subsection{Type of Hepatitis B Infection (Acute or Chronic)}

Hepatitis B is said to be acute if it lasts for less than 6 months or chronic if it lasts for more than 6 months [4]. The distinction between these 2 types of infection can be made by relying on the presence of demonstrable antibodies to the core antigen [14]. Acute hepatitis B is suspected if there are detectable IgM antibodies to the Hep B core Ag, while a presence of IgG antibodies is indicative of Chronic Hepatitis B.

In our study $33.3 \%$ of those actively infected with HBV were positive for the HepB core IgM, 66.7\% had negative HBcAb IgM but positive $\mathrm{HBcAb}$ IgG an indication of chronic hepatitis B infection [14]. In a cohort study with over 1.7 million individuals done in British Colombia from 1990-2015 describing the characteristics of people diagnosed with acute and chronic hepatitis $B$ virus (HBV) infection, $4.3 \%$ of the individual had acute Hepatitis B infection and 95.7\% of the individuals had chronic infection [15]. In both studies high values were reported for chronic infection however, the difference between our findings and those from the Colombian study may be because our study was a cross-sectional and sample size was 254 participants. Our findings are reflective 
of what is happening elsewhere in that many individuals have chronic hepatitis $\mathrm{B}$ [15]. The greatest (95\%) transmission of $\mathrm{HBV}$ is during perinatal period, $30 \%$ in children below the age of 5 [2]. Most of these infections result in the development of chronic HepB. On the other hand, only 5\% of healthy adults infected with HBV will develop chronic HepB [16]; therefore, a high chronic hepatitis B is reflective of perinatal and early childhood transmission.

We cannot conclusively deduce that $33.3 \%$ of our individuals had acute infection. Hepatitis B core antibody IgM is detectable in both acute viral Hepatitis B and acute-on-chronic HepB reactivation [17]. Quantification of HBcAb IgM is useful to distinguish acute viral hepatitis $B$ infections from acute on chronic HepB reactivation [18]. However, this was not done in this study. Another effective way to determine individuals with chronic hepatitis B is to repeat HBsAg test after six months [14].

\subsection{Evaluation of Infectiousness HBV among HBsAg Positive Individuals}

Evaluation of infectiousness of HBV is determined based on the detection of the envelope antigen [14] and viral load above 20,000 IU/mL [5] both of which reflect active viral replication.

In this study, none of the HBsAg positive individuals had a demonstrable Hepatitis $B$ envelop antigen, $33 \%$ of chronic Hepatitis B individuals had a high viral load above 20,000 IU/mL but with a negative HBeAg. Such individuals, have been reported to harbor a mutant form of $\mathrm{HBV}$ that does not produce $\mathrm{HBeAg}$, due to a mutation in the precore or core promoter region [16], alternatively, patients may seroconvert, through development of HepBeAb. In addition, a study done in Singapore about chronic HBV infection in pregnancy revealed that spontaneous $\mathrm{HBeAg}$ seroconversion among $\mathrm{HBeAg}$ positive pregnant women occur during peripartum period yet the mother continue having a high viral load [19]. Therefore, it is plausible to believe that our HBsAg positive individual with viral load > 20,000 IU/mL had $\mathrm{HBeAg}$ seroconversion during peripartum or a mutant HBV. Hence, determining HBV viral load would be more important in determining $\mathrm{HBV}$ infectiousness as compared to testing $\mathrm{HBeAg}$.

\subsection{Evaluation of Immunity}

Immunity to hepatitis B can be acquired naturally; (when an individual suffers from Hepatitis B and develops antibodies to the surface antigen) or artificially through vaccination [4].

In this study, it was observed that $14.6 \%$ tested $\mathrm{HBs} A \mathrm{~b}$ positive (a marker for HBV immunity) and majority of these (72\%) had acquired immunity to HBV naturally. This implies that there is a high rate of transmission of $\mathrm{HBV}$ especially among adults but because they have a competent immune system, they are able to develop antibodies that clear the infection [3] and that very few individuals are immune as a result of being vaccinated. The findings of a high rate of transmission have implications, especially given the role of Hepatitis B in the causa- 
tion of Cancer of the liver. Suffice to note that HBV integrates and persists for life in the hepatocyte nuclei as covalently closed circular DNA (cccDNA) [4] thus the virus may reactivate when HBsAb levels reduce [11]; moreover the integrated virus accelerates procarcinogenic events that trigger hepatocyte turnover [3] and may lead to cancer of the liver. Our findings are different from what was observed among Health Care Workers (HCWs) in Indonesia where 18.5\% had natural immunity and $36 \%$ had artificial immunity [20]. This difference is expected and it would probably be observed in our study had it been done among HCWs in whom HepB vaccination is offered before one starts to interface with patients. Furthermore, unlike in Uganda where it started in 2012 [6] HepB vaccination in many developed countries has been a routine for decades, therefore most individuals have artificial immunity, taken together, these results imply that there is a low uptake of Hepatitis B vaccine and a high transmission of HBV among adults. The only effective way to reduce HBV infection among adults, is to test and actively immunize individuals who are found HBsAg negative while those found HBsAg positive, HBV monitoring and treatment should be initiated [5]. In children; babies born to $\mathrm{HBsAg}$ and $\mathrm{HBeAg}$ positive mothers Hepatitis B immunoglobulins together with $\mathrm{HepB}$ vaccine should be given within 24 hours of birth; on the other hand, babies born to HBsAg positive yet $\mathrm{HBe} A g$ negative should be given HepB vaccine within 24 hours of birth [5].

What's more, the presence of antibodies to the HBsAg its self is not an indication of sufficient protection from HBV re-infection or reactivation. Sufficient protection to $\mathrm{HBV}$ is observed when $\mathrm{HBs} A b$ titers are greater than $10 \mathrm{mIU} / \mathrm{mL}$ [5]. In this study, we observed that $11 \%$ of those who were $\mathrm{HBsAb}$ positive did not have sufficient immunity ( $\mathrm{HBsAb}<10 \mathrm{mIU} / \mathrm{ml}$ ) and the majority $(75 \%)$ had acquired immunity following artificial vaccination. Findings from a study in $\mathrm{Pa}$ kistan reported that among the vaccinated children immunity dropped to $39.8 \%$ between the age of 7 - 19 but improved when a booster dose was administered [21]. In this study, it was not possible to verify when the vaccine was given or the quality of the vaccine that was administered, all of which affect the level of immunity after artificial vaccination, alternatively, it's a possibility that the participants had recently been vaccinated, thus they need time to build sufficient immunity, which warrants follow up evaluation. In general, these findings, therefore, indicate the need to perform HBsAb quantification test so as to determine whether those vaccinated have sufficient immunity.

\section{Study Limitations}

This study was carried out on pregnant women attending ANC at lower health centers and thus the results may not be applied to higher health centers e.g referrals and private hospitals.

Majority of participants were from Mbarara District, there is a need to assess the situation in other districts.

A quantification $\mathrm{HBcAb}$ IgM test was not done, thus the conclusion of either acute viral infection or acute on chronic reactivation was not possible. 


\section{Conclusions}

- This study has found that the prevalence of Hepatitis B among pregnant women at lower health centers in Mbarara municipality is $1.2 \%$.

- Of those infected with HBV, $67 \%$ had chronic hepatitis B while $33 \%$ had acute viral hepatitis $B$ infection or acute on chronic hepatitis $B$ reactivation.

- There was no demonstrable $\mathrm{HBeAg}$ among $\mathrm{HBsAg}$ positive pregnant women, however, HBV viral load above $20,000 \mathrm{IU} / \mathrm{mL}$ was observed in $33 \%$; therefore, when determining infectiousness one needs to assay for both $\mathrm{HBeAg}$ and HBV viral load.

- There are many individuals (84.2\%) at risk of HBV infection, since they lack protective antibodies in blood

- $14.6 \%$ of pregnant women are immune to hepatitis either following recovery from a natural exposure (10.2\%) or after receiving a vaccine (3.9\%). Of those with positive $\mathrm{HBsAb}, 11 \%$ had insufficient immunity ( $\mathrm{HBsAb}<10 \mathrm{mIU} / \mathrm{mL}$ ) to $\mathrm{HBV}$ and $75 \%$ of these had acquired immunity by artificial vaccination.

\section{Recommendations}

1) A quantification test of $\mathrm{HBcAb} \operatorname{IgM}$ to distinguish between acute viral hepatitis $B$ and acute chronic hepatitis $B$ reactivation.

2) Genotyping of $\mathrm{HBV}$ among $\mathrm{HBeAg}$ negative to evaluate the role of mutation

3) Since many pregnant women were not vaccinated against hepatitis B yet there was a significant transmission, efforts should be put in place to vaccinate the community of Mbarara Municipality

4) Evaluate the levels of HBsAb especially among of the artificial immunity, so that booster vaccination is offered.

5) Routine HBsAg testing of pregnant women during antenatal visits because of the asymptomatic nature of HBV.

\section{Acknowledgements}

The authors are grateful to:

The Kayanja Fellowship at Mbarara University of Science and Technology for sponsoring the study.

The participants for their participation.

Mbarara Municipal Council for granting permission to use their Health Centers.

Dr. Kumbakumba Elias the Paediatrician on the study.

Kyarimpa Rose and Sophie Kobugabe research assistants.

Dr. Male Kenneth the independent observer of the study.

Uganda National Health Laboratory Services-Hepatitis B section.

MBN Clinical laboratories.

\section{Conflicts of Interest}

The authors declare no conflicts of interest regarding the publication of this paper. 


\section{References}

[1] WHO (2017) Global Hepatitis Report. France.

[2] Burns, G.S. and Thompson, A.J. (2014) Viral Hepatitis B: Clinical and Epidemiological Characteristics. Cold Spring Harbor Perspectives in Medicine, 4, a024935. https://doi.org/10.1101/cshperspect.a024935

[3] Chisari, F.V., Isogawa, M. and Wieland, S.F. (2010) Pathogenesis of Hepatitis B Virus Infection. Pathologie Biologie, 58, 258-266. https://doi.org/10.1016/j.patbio.2009.11.001

[4] Mauss, S., et al. (2017) Hepatology. 8th Edition, Druckerei Heinrich GmbH, German.

[5] WHO (2015) Guidelines for the Prevention, Care and Treatment of Persons with Chronic Hepatitis B Infection. France.

[6] UNEPI (2013) Uganda National Expanded Programme on Immunization Multi Year Plan 2012-2016. Kampala.

[7] UPHIA (2017) Uganda Population-Based HIV Impact Assessment (UPHIA), in Summary Sheet: Preliminary Findings August 2017. Ministry of Health, Kampla, Uganda.

[8] Bayo, P., et al. (2014) High Prevalence of Hepatitis B virus Infection among Pregnant Women Attending Antenatal Care: A Cross-Sectional Study in two Hospitals in Northern Uganda. BMJ Open, 4, e005889.

https://doi.org/10.1136/bmjopen-2014-005889

[9] Caligiuri, P., et al. (2016) Overview of Hepatitis B virus Mutations and Their Implications in the Management of Infection. World Journal of Gastroenterology, 22, 145-154. https://doi.org/10.3748/wjg.v22.i1.145

[10] Hall, A.J. (2006) Immunisation against Infectious Disease. The Stationery Office, UK.

[11] Tien, Y.-C., et al. (2018) Changes in Hepatitis B virus Surface Antibody Titer and Risk of Hepatitis B Reactivation in HBsAg-Negative/HBcAb-Positive Patients Undergoing Biologic Therapy for Rheumatic Diseases: A Prospective Cohort Study. Arthritis Research \& Therapy, 20, 246. https://doi.org/10.1186/s13075-018-1748-Z

[12] Israel, G.D. (2003) Determining sample size, A.E.a. communication, Editor., PEOD6: USA.

[13] Råssjö, E., Mirembe, F. and Darj, E. (2011) Self-Reported Sexual Behaviour among Adolescent Girls in Uganda: Reliability of Data Debated. African Health Sciences, 11, 383-389.

[14] Mahy, B. and Van Regenmortel, M. (2010) Desk Encyclopedia of Human and Medical Virology. In: Mahy, B. and Van Regenmortel, M., Eds., Encyclopedia of Virolo$g y$, Elsevier Ltd., CA, USA.

[15] Binka, M., et al. (2018) Differing Profiles of People Diagnosed with Acute and Chronic Hepatitis B virus Infection in British Columbia, Canada. World Journal of Gastroenterology, 24, 13.

[16] Zhang, Z.H., et al. (2016) Genetic Variation of Hepatitis B virus and its Significance for Pathogenesis. World Journal of Gastroenterology, 22, 126-144. https://doi.org/10.3748/wjg.v22.i1.126

[17] Park, J.W., et al. (2015) Differentiation of Acute and Chronic Hepatitis B in IgM Anti-HBc Positive Patients. World Journal of Gastroenterology, 21, 3953-3959. https://doi.org/10.3748/wjg.v21.i13.3953

[18] Puri, P. (2013) Acute Exacerbation of Chronic Hepatitis B: The Dilemma of Diffe- 
rentiation from Acute Viral Hepatitis B. Clinical and Experimental Hepatology, 3, 301-312. https://doi.org/10.1016/j.jceh.2013.08.014

[19] Tan, H.-H., Lui, H.-F. and Chow, W.-C. (2008) Chronic Hepatitis B Virus (HBV) Infection in Pregnancy. Hepatology International, 2, 370-375.

https://doi.org/10.1007/s12072-008-9063-4

[20] Muljono, D.H., Wijayadi, T. and Sjahril, R. (2018) Hepatitis B Virus Infection among Health Care Workers in Indonesia. Euroasian Journal of Hepato-Gastroenterology, 8, 88-92. https://doi.org/10.5005/jp-journals-10018-1269

[21] Qawasmi, M., et al. (2015) Age-Dependent Decrease of Anti-HBs Titers and Effect of Booster Doses Using 2 Different Vaccines in Palestinian Children Vaccinated in Early Childhood. Human Vaccines \& Immunotherapeutics, 11, 1717-1724.

https://doi.org/10.1080/21645515.2015.1041687 